\title{
Approximation algorithms for the $p$-hub center routing problem in parameterized metric graphs ${ }^{\star}$
}

\author{
Li-Hsuan Chen ${ }^{1}$, Sun-Yuan Hsieh ${ }^{2}$, Ling-Ju Hung ${ }^{1}$, Ralf Klasing ${ }^{3}$ \\ 1 AROBOT Innovation CO., LTD, New Taipei City 235, Taiwan \\ \{lihsuan. chen, lingju. hung\} @arobot. info \\ 2 Department of Computer Science and Information Engineering \\ National Cheng Kung University, Tainan 701, Taiwan \\ hsiehsy@mail.ncku.edu.tw \\ ${ }^{3}$ CNRS, LaBRI, Université de Bordeaux \\ 351 Cours de la Libération, 33405 Talence cedex, France \\ ralf.klasing@labri.fr
}

\begin{abstract}
A complete weighted graph $G=(V, E, w)$ is called $\Delta_{\beta}$ metric, for some $\beta \geq 1 / 2$, if $G$ satisfies the $\beta$-triangle inequality, i.e., $w(u, v) \leq \beta \cdot(w(u, x)+w(x, v))$ for all vertices $u, v, x \in V$. Given a $\Delta_{\beta}$-metric graph $G=(V, E, w)$, the Single Allocation at most $p$ Hub Center Routing problem is to find a spanning subgraph $H^{*}$ of $G$ such that (i) any pair of vertices in $C^{*}$ is adjacent in $H^{*}$ where $C^{*} \subset V$ and $\left|C^{*}\right| \leq p$; (ii) any pair of vertices in $V \backslash C^{*}$ is not adjacent in $H^{*}$; (iii) each $v \in V \backslash C^{*}$ is adjacent to exactly one vertex in $C^{*}$; and (iv) the routing cost $r\left(H^{*}\right)=\sum_{u, v \in V} d_{H^{*}}(u, v)$ is minimized where $d_{H^{*}}(u, v)=w\left(u, f^{*}(u)\right)+w\left(f^{*}(u), f^{*}(v)\right)+w\left(v, f^{*}(v)\right)$ and $f^{*}(u), f^{*}(v)$ are the vertices in $C^{*}$ adjacent to $u$ and $v$ in $H^{*}$, respectively. Note that $w\left(v, f^{*}(v)\right)=0$ if $v \in C^{*}$. The vertices selected in $C^{*}$ are called hubs and the rest of vertices are called non-hubs. In this paper, we show that the Single Allocation at most $p$-Hub Center Routing problem is NP-hard in $\Delta_{\beta}$-metric graphs for any $\beta>1 / 2$. Moreover, we give $2 \beta$ approximation algorithms running in time $O\left(n^{2}\right)$ for any $\beta>1 / 2$ where $n$ is the number of vertices in the input graph.
\end{abstract}

\section{Introduction}

The design of hub-and-spoke networks is a key issue with applications in transportation, e.g., airlines [21] and cargo delivery systems [31]. The major concern to design a hub-and-spoke network with high quality is to connect a large amount of origin/destination (O/D) pairs by using a small number of links. The usage of hub facilities helps to reduce the connections between all nodes. To locate $p$

\footnotetext{
* This study has been carried out in the frame of the "Investments for the future" Programme IdEx Bordeaux - CPU (ANR-10-IDEX-03-02). Research supported by the LaBRI under the "Projets émergents" program. The main work for this article was done while Li-Hsuan Chen and Ling-Ju Hung (corresponding author) were with the National Cheng Kung University.
} 
hubs in hub networks in order to route the traffic between origin/destination pairs with minimum cost is the classical hub location problem called the $p$-HUB MEDIAN problem $[30,32]$. Notice that the general $p$-hub median problem considers that each pair of origin/destination has different unit traffic (flow) cost. We call a hub location problem multi-allocation, if a demand node can be served by several hubs. If each demand node can be served by exactly one hub, the hub location problem is single-allocation. The $p$-hub median problem is NP-hard. Many linear programming-based and heuristic algorithms were proposed to solve the $p$-hub median problem and its variants (see the survey papers $[1,14,28]$ ).

Another hub location problem, the Single Allocation $p$-Hub Center problem, is to choose a fixed number $p$ of vertices as hubs and to assign each nonhub vertex to exactly one of the chosen hubs in such a way that the maximum distance/cost between origin-destination pairs is minimized [13,31]. Unlike the $p$-HuB MEDIAN problem to minimize the total cost of all origin-destination pairs, the Single Allocation $p$-Hub Center problem is to minimize the poorest service quality. Chen et al. [15] proved that for any $\epsilon>0$, it is NPhard to approximate the Single Allocation $p$-Hub Center problem to a ratio $\frac{4}{3}-\epsilon$ and gave a $\frac{5}{3}$-approximation algorithm running in time $O\left(p n^{3}\right)$ to solve the same problem. If the input graph is $\Delta_{\beta}$-metric, it was proved that for any $\varepsilon>0$, to approximate the Single Allocation $p$-Hub Center problem to a ratio $g(\beta)-\varepsilon$ is NP-hard where $g(\beta)$ is a function of $\beta$ and a series of $r(\beta)$-approximation algorithms were given in [18] where $r(\beta)$ is a function of $\beta$. The STAR $p$-Hub CENTER problem is another hub location problem with minmax criterion. It is to pick $p$ nodes as hubs among the set of demand nodes connecting with the central given hub $c$ and to connect each of the remaining demand nodes to exactly one of the $p$ chosen hubs such that the longest path in the tree structure network is minimized. Chen et al. [16] showed that for any $\epsilon>0$, to approximate the STAR $p$-HUB CENTER problem to a ratio $1.5-\epsilon$ is NPhard and gave a $\frac{5}{3}$-approximation algorithms for the same problem. Moreover, for input graphs satisfying $\beta$-triangle inequality, i.e., $w(u, v) \leq \beta \cdot(w(u, x)+w(x, v))$ for all vertices $u, v, x$ in the input graph $G=(V, E, w)$ and $\beta \geq 1 / 2$, it was shown that for any $\epsilon>0$, to approximate the STAR $p$-HuB CENTER problem to a ratio $g(\beta)-\epsilon$ is NP-hard and $r(\beta)$-approximation algorithms were given in the same paper where $g(\beta)$ and $r(\beta)$ are functions of $\beta[17,19]$.

Despite numerous research results on solving various hub location problems in the past twenty-five years [14,20], the design of approximation algorithms for hub location problems only made very little progress in the past two decades, especially for the $p$-HUB MEDIAN problem $[25,26]$. In this paper, we consider a variant of the $p$-HUB MEDIAN problem in which each pair of origin/destination has the same unit traffic (flow) cost called the Single Allocation AT most $p$-Hub Center Routing problem. The Single Allocation at most $p$-Hub Center Routing problem is to choose at most $p$ vertices as hubs and to assign each remaining vertex (called non-hub) to exactly one of the chosen hubs in such a way that the sum of distance/cost between all origin-destination pairs is minimized, i.e., the routing cost is minimized. There are some routing cost 
optimization problems on finding a spanning subtrees or a spanning tree satisfying certain properties of the input graph such that the routing cost is minimized $[27,33,34]$. Some of these minimum routing cost spanning tree problems admit polynomial-time approximation schemes [33,34].

Our study uses the well-known concept of stability of approximation for hard optimization problems $[9,11,22,23]$. The idea of this concept is similar to that of the stability of numerical algorithms. But instead of observing the size of the change in the output value according to a small change of the input value, one is interested in the size of the change of the approximation ratio according to a small change in the specification (some parameters, characteristics) of the set of problem instances considered. If the change of the approximation ratio is small for every small change in the set of problem instances, then the algorithm is called stable. The concept of stability of approximation has been successfully applied to several fundamental hard optimization problems. E.g. in [2-4, 8-10, $12,29]$ it was shown that one can partition the set of all input instances of the Traveling Salesman Problem into infinitely many subclasses according to the degree of violation of the triangle inequality, and for each subclass one can guarantee upper and lower bounds on the approximation ratio. Similar studies demonstrated that the $\beta$-triangle inequality can serve as a measure of hardness of the input instances for other problems as well, in particular for the problem of constructing 2-connected spanning subgraphs of a given complete edge-weighted graph [5], and for the problem of finding, for a given positive integer $k \geq 2$ and an edge-weighted graph $G$, a minimum $k$-edge- or $k$-vertex-connected spanning subgraph $[6,7]$.

In this paper, we consider a graph $G=(V, E, w)$ with a distance function $w(\cdot, \cdot)$ being a $\Delta_{\beta}$-metric graph on $V$ such that $w(v, v)=0, w(u, v)=w(v, u)$, and $w(u, v) \leq \beta \cdot(w(u, x)+w(x, v))$ for all $u, v, x \in V$. Given a positive integer $p$, let $H^{*}$ be a spanning subgraph of $G$ satisfying the conditions that vertices (hubs) in $C^{*} \subset V$ form a clique of size at most $p$ in $H^{*}$, vertices (non-hubs) in $V \backslash C^{*}$ form an independent set in $H^{*}$, and each non-hub $v \in V \backslash C^{*}$ is adjacent to exactly one hub in $C^{*}$. Define $d_{H^{*}}(u, v)=w\left(u, f^{*}(u)\right)+w\left(f^{*}(u), f^{*}(v)\right)+w\left(v, f^{*}(v)\right)$ where $f^{*}(u)$ and $f^{*}(v)$ are hubs adjacent to $u$ and $v$ in $H^{*}$ respectively. Notice that if $u$ is a hub in $H^{*}$ then $w\left(u, f^{*}(u)\right)=0$. Let $r\left(H^{*}\right)=\sum_{u, v \in V} d_{H^{*}}(u, v)$ be the routing cost of $H^{*}$. We list the formal definition of the SingLE ALLOCATION AT most $p$-Hub Center Routing problem in the following.

Single Allocation at most $p$-Hub Center Routing $\left(\Delta_{\beta}\right.$-SA $\left.p \mathrm{HCR}\right)$

Input: $\quad \mathrm{A} \Delta_{\beta}$-metric graph $G=(V, E, w)$ and a positive integer $p$.

Output: A spanning subgraph $H^{*}$ of $G$ satisfying the following conditions (i) any pair of vertices (hubs) in $C^{*}$ is adjacent in $H^{*}$ where $C^{*} \subset V$ and $\left|C^{*}\right| \leq p$; (ii) any pair of vertices (non-hubs) in $V \backslash C^{*}$ is not adjacent in $H^{*}$; (iii) each non-hub $v \in V \backslash C^{*}$ is adjacent to exactly one hub in $C^{*}$ such that $r\left(H^{*}\right)$ is minimized.

In Fig. 1, we give an example of the Single Allocation AT MOst $p$-HuB CEnter Routing problem that can be applied in the design of post mail networks for which hubs are major post offices and non-hubs are small post offices. 


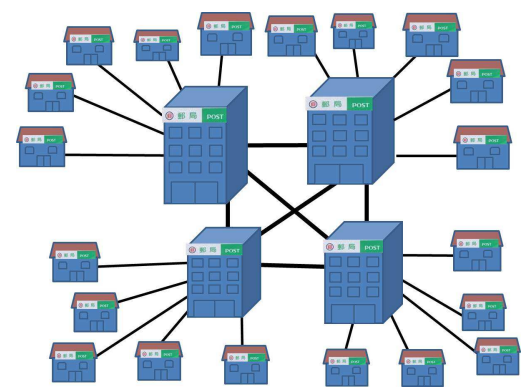

Fig. 1. An example of a single allocation at most $p$-hub center routing network where the four hubs are the major post offices and the non-hubs are the other small post offices.

In this paper, we investigate the approximability of the Single Allocation AT most $p$-Hub Center Routing problem in $\Delta_{\beta}$-metric graphs. The paper is organized as follows: In Section 2, we prove that the Single Allocation AT most $p$-Hub Center Routing problem is NP-hard in $\Delta_{\beta}$-metric graphs for any $\beta>1 / 2$. In Section 3 , for any $\beta>1 / 2$, we give $2 \beta$-approximation algorithms running in time $O\left(n^{2}\right)$ for the Single Allocation at most $p$-Hub Center RouTing problem.

\section{NP-hardness}

In this section, we show that for any $\beta>1 / 2$, the Single Allocation AT most $p$-Hub Center Routing problem is NP-hard.

Theorem 1. For any $\beta>1 / 2$, the Single Allocation at most $p$-Hub CenTER Routing problem in $\Delta_{\beta}$-metric graphs is NP-hard.

Proof. We prove that the Single Allocation at most $p$-Hub Center RoutING problem is at least as hard as the well-known NP-hard problem MAXIMUM Clique [24].

Maximum Clique Problem [24]

Input: A simple undirected graph $G=(V, E)$ and a positive integer $k$. Output: Whether there is a clique $S \subseteq V$ of size $k$ in $G$.

Notice that if $G$ has a universal vertex $v$, i.e., $\operatorname{deg}_{G}(v)=|V|-1$, then we can simply select $v$ in $S$ and ask whether there exists a size $k-1$ clique in $G[V \backslash\{v\}]$. Thus, we may assume that $G$ has no universal vertex. To show such a statement, we reduce the input $G=(V, E)$ of the Maximum Clique problem to the Single Allocation at most $p$-Hub Center Routing problem where $p=k+1$. According to $G$, we construct an input $\Delta_{\beta}$-metric graph $G^{\prime}=\left(V^{\prime}, E^{\prime}, w\right)$ where $V^{\prime}=V \cup\{x\}, E^{\prime}=\left\{(u, v) \mid u, v \in V^{\prime}\right\}$, and assign the cost of each edge in $E^{\prime}$ as follows. 
$-w(u, v)=1$ if $(u, v)$ is an edge in $G$.

- $w(u, v)=1+\epsilon$ if $(u, v)$ is a non-edge in $G$ where $0<\epsilon<1$.

$-w(x, v)=1$ for all $v \in V$.

It is not hard to see that $G^{\prime}$ is a $\Delta_{\beta}$-metric graph for any $\beta \geq \frac{1+\epsilon}{2}$. Notice that for any constant $\beta$, it defines a $\Delta_{\beta}$-metric graph class and this graph class contains all $\Delta_{\beta^{\prime}}$-metric graphs for $\beta^{\prime} \leq \beta$. If one can prove for any $1 / 2<\beta<1$ the Single Allocation at most $p$-Hub Center Routing problem is NP-hard, then it implies for any $\beta>1 / 2$ this problem is NP-hard. In the following proof, we may assume that $1 / 2<\beta<1$.

Let $H^{*}$ be an optimal solution of the Single Allocation AT most $p$-Hub Center Routing problem. Let $S^{*}$ be a size $k$ clique in $G$. We then obtain a solution $H$ of the Single Allocation at most $p$-Hub Center Routing problem by letting all vertices $C=S^{*} \cup\{x\}$ be the set of hubs in $H$ and letting all the remaining vertices in $V \backslash S^{*}$ be non-hubs adjacent to $x$. We obtain the following facts.

- For two non-hubs $y, z, \operatorname{dist}_{H}(y, z)=2$.

- For a hub $v \in C \backslash\{x\}$ and a non-hub $y, \operatorname{dist}_{H}(v, y)=2$.

- For two hubs $u, v \in C, \operatorname{dist}_{H}(u, v)=w(u, v)$.

- For any vertex $v \in V, \operatorname{dist}_{H}(v, x)=w(v, x)=1$.

We see that the routing cost of $H$ is $r(H)=2 \cdot\left(\begin{array}{l}n \\ 2\end{array}\right)-\left(\begin{array}{c}k \\ 2\end{array}\right)+n=n^{2}-\left(\begin{array}{c}k \\ 2\end{array}\right)$ where $n=|V|$.

Since $H^{*}$ is an optimal solution of the Single Allocation at most $p$-Hub Center Routing problem in $G^{\prime}$, we have $r\left(H^{*}\right) \leq n^{2}-\left(\begin{array}{c}k \\ 2\end{array}\right)$.

Claim 1. All non-hubs in $H^{*}$ must be adjacent to the same hub.

Proof. Suppose that there are at least two hubs adjacent to non-hubs. Then the routing cost between any two non-hubs which are adjacent to different hubs is at least 3 . This will imply that $r\left(H^{*}\right) \geq\left(\frac{3-\rho}{2}\right) \cdot n^{2}-\left(\begin{array}{c}k \\ 2\end{array}\right)>n^{2}-\left(\begin{array}{c}k \\ 2\end{array}\right)$ where $0<\rho<1$, a contradiction to the assumption that $H^{*}$ is an optimal solution of the SingLE Allocation at most $p$-Hub Center Routing.

Claim 2. The number of hubs in $H^{*}$ is $p$.

Proof. If the number of hubs is less than $p$, i.e., $\left|C^{*}\right|<p$, then we may obtain another solution $H^{\prime}$ by selecting $p-\left|C^{*}\right|$ non-hubs in $H^{*}$ and let them be hubs in $H^{\prime}$. Since all hubs are pairwise adjacent and $\beta<1$, it is not hard to see that $r\left(H^{\prime}\right)<r\left(H^{*}\right)$. It contradicts the assumption that $H^{*}$ is an optimal solution of the Single Allocation at most $p$-Hub Center Routing. This shows that the number of hubs in $H^{*}$ must be $p$.

Claim 3. The vertex $x$ must be a hub in $H^{*}$.

Proof. Suppose that $x$ is not a hub. Since $G$ has no universal vertex, in $H^{*}$ the hub which is adjacent to all non-hubs must be incident to some edges with edge cost $1+\epsilon$. We see that $r\left(H^{*}\right) \geq\left(\frac{2+\epsilon^{\prime}}{2}\right) \cdot n^{2}-\left(\begin{array}{c}k \\ 2\end{array}\right)>n^{2}-\left(\begin{array}{c}k \\ 2\end{array}\right)=r(H)$ where $\epsilon^{\prime}>0$, a contradiction to the assumption that $H^{*}$ is an optimal solution of the 
Single Allocation at most $p$-Hub Center Routing. This completes the proof that $x$ must be a hub in $H^{*}$.

Claim 4. If the Single Allocation at most $p$-Hub Center Routing has an optimal solution $H^{*}$ with $r\left(H^{*}\right)=n^{2}-\left(\begin{array}{c}k \\ 2\end{array}\right)$ and $C^{*}$ is the set of hubs in $H^{*}$, then $C^{*} \backslash\{x\}$ is a clique of size $k$ in $G$ where $k=p-1$.

Proof. According to Claims 1-3 that all non-hubs are adjacent to $x$ and $\left|C^{*}\right|=$ $p=k+1$, we have the routing cost between vertices in $C^{*}$ is

$$
\begin{aligned}
r\left(C^{*}\right) & =r\left(H^{*}\right)-r\left(V^{\prime} \backslash C^{*}\right)-r\left(V^{\prime} \backslash C^{*}, C^{*}\right) \\
& =\left(n^{2}-\left(\begin{array}{c}
k \\
2
\end{array}\right)\right)-2 \cdot\left(\begin{array}{c}
n-k \\
2
\end{array}\right)-((n-k)+2 \cdot k(n-k))=\left(\begin{array}{c}
k+1 \\
2
\end{array}\right) .
\end{aligned}
$$

Notice that $w(x, v)=1$ for $v \in V, w(u, v)=1$ if $(u, v) \in E$, otherwise $w(u, v)=1+\epsilon$. Since $r\left(C^{*}\right)=\sum_{u, v \in C^{*}} w(u, v)=\left(\begin{array}{c}k+1 \\ 2\end{array}\right)$, we see that for $u, v \in$ $C^{*} \backslash\{x\}, w(u, v)=1$ and $C^{*} \backslash\{x\}$ forms a clique in $G$.

According to Claim 4, if there exists a polynomial time algorithm that solves Single Allocation at most $p$-Hub Center Routing with routing cost $n^{2}-\left(\begin{array}{l}k \\ 2\end{array}\right)$ where $k=p-1$, then the MaXimum Clique problem can be solved in polynomial time. However, MAXIMUM CLIQUe is a well-known NP-hard problem [24]. By the fact Maximum Clique is an NP-hard problem, this implies that Single Allocation at most $p$-Hub Center Routing is also an NPhard problem.

\section{New approximation algorithms}

We have shown the NP-hardness of the Single Allocation at most $p$-Hub Center Routing problem by reducing from the maximum clique problem. It is well-known that the maximum clique is hard to approximate. In this section, we give approximation algorithms for the Single Allocation at most $p$-Hub Center Routing problem.

We first state a property of $\Delta_{\beta}$-metric graphs in the following lemma.

Lemma 1 ([8]). Let $G=(V, E)$ be a $\Delta_{\beta}$-metric graph for $\frac{1}{2} \leq \beta<1$. For any two edges $(u, x),(v, x)$ with a common endvertex $x$ in $G, w(u, x) \leq \frac{\beta}{1-\beta} \cdot w(v, x)$.

Theorem 2. For any $1 / 2 \leq \beta \leq 1$, there is a $2 \beta$-approximation algorithm for the Single Allocation at most $p$-Hub Center Routing problem.

Proof. It is easy to see that in time $O\left(n^{2}\right)$, Algorithm 1 returns a feasible solution of the Single Allocation at most $p$-Hub Center Routing problem. We now prove that the solution $H$ returned by Algorithm 1 satisfies the approximation ratio $2 \beta$. Let $G=(V, E, w)$ be the input graph of the Single AlloCATION at most $p$-Hub Center Routing problem. Let $H^{*}$ be an optimal solution of the Single Allocation at most $p$-Hub Center Routing problem. Let $C^{*}$ denote the set of hubs in $H^{*}$. Define $w\left(H^{*}\right)=\sum_{(u, v) \in E\left(H^{*}\right)} w(u, v)$. 
Algorithm 1: Approximation algorithm for $\Delta_{\beta}$-SA $p$ HCR for $1 / 2 \leq \beta \leq 1$

Let $U:=V$. Initially, $C=\emptyset$. Construct a spanning subgraph $H$ of $G$ by the following steps.

Step 1: Find $z=\arg \min _{v \in V} \sum_{u \in V} w(u, v)$ as a hub in $H$.

Step 2: Pick $p-1$ vertices $\left\{v_{1}, \ldots, v_{p-1}\right\}$ farthest to $z$ from $U$. Let $C:=C \cup$ $\left\{z, v_{1}, \ldots, v_{p-1}\right\}$ be the set of hubs in $H$ and $U:=U \backslash\left\{z, v_{1}, \ldots, v_{p-1}\right\}$.

Step 3: Connect all vertices in $U$ to $z$ as non-hubs in $H$.

Step 4: Return $H$.

Construct a weighted complete graph $G^{*}=\left(V, E, w^{*}\right)$ according to $H^{*}$ where $w^{*}(u, v)=\operatorname{dist}_{H^{*}}(u, v)$. Define

$$
w^{*}\left(G^{*}\right)=\sum_{u, v \in V} w^{*}(u, v)=\sum_{u, v \in V} \operatorname{dist}_{H^{*}}(u, v)=r\left(H^{*}\right) .
$$

Let $H$ be the solution returned by Algorithm 1 with $z$ being the only hub in $H$ that is adjacent to non-hubs. Let $S_{z}$ be the spanning star of $G$ with center $z$ satisfying $z=\arg \min _{v \in V} \sum_{u \in V} w(u, v)$. We use $f^{*}(z)$ to denote the hub adjacent to $z$ in $H^{*}$. Note that $f^{*}(z)=z$ if $z$ is a hub in $H^{*}$. Let $S_{v}$ be the spanning star of $G^{*}$ with center $v$ and $w^{*}\left(S_{v}\right)=\sum_{(u, v) \in E\left(S_{v}\right)} w^{*}(u, v)$.

Claim 1. $r(H) \leq r\left(S_{z}\right)-(1-\beta) \cdot \sum_{u, v \in C \backslash\{z\}}(w(z, u)+w(z, v))$

Proof. According to the $\beta$-triangle inequality for $u, v \in V, w(u, v) \leq \beta(w(z, u)+$ $w(z, v))$. We obtain that

$$
\begin{aligned}
r(H) & =r\left(S_{z}\right)-\sum_{u, v \in C \backslash\{z\}}(w(z, u)+w(z, v)-w(u, v)) \\
& \leq r\left(S_{z}\right)-(1-\beta) \cdot \sum_{u, v \in C \backslash\{z\}}(w(z, u)+w(z, v)) .
\end{aligned}
$$

This completes the proof.

Claim 2. $\sum_{u, v \in C^{*} \backslash\left\{f^{*}(z)\right\}} w(u, v) \leq \sum_{u, v \in C \backslash\{z\}}(w(z, u)+w(z, v))$

Proof. We obtain that

$$
\begin{aligned}
\sum_{u, v \in C^{*} \backslash\left\{f^{*}(z)\right\}} w(u, v) \leq & \sum_{\substack{u, v \in C^{*} \backslash\left\{f^{*}(z)\right\}\\
}} \beta \cdot(w(z, u)+w(z, v)) \\
\leq & \sum_{\substack{u, v \in C \backslash\{z\} \\
\text { (since the selection of hubs in Algorithm 1) }}} \beta \cdot(w(z, u)+w(z, v)) \\
& \leq \sum_{u, v \in C \backslash\{z\}}(w(z, u)+w(z, v)) .
\end{aligned}
$$


This completes the proof.

Now we prove $r(H) \leq 2 \beta \cdot r\left(H^{*}\right)$ in the following.

$$
\begin{aligned}
& r\left(H^{*}\right)=w^{*}\left(G^{*}\right)=\frac{1}{2} \cdot \sum_{v \in V} w^{*}\left(S_{v}\right) \\
& \geq \frac{1}{2 \beta} \cdot \sum_{v \in V} w\left(S_{v}\right)-\left(\frac{1-\beta}{2 \beta}\right) \cdot \sum_{(u, v) \in E\left(H^{*}\right)} w^{*}(u, v) \\
& =\frac{1}{2 \beta} \cdot \sum_{v \in V} w\left(S_{v}\right)-\left(\frac{1-\beta}{2 \beta}\right) \cdot \sum_{(u, v) \in E\left(H^{*}\right)} w(u, v) \\
& =\frac{1}{2 \beta} \cdot \sum_{v \in V} w\left(S_{v}\right)-\left(\frac{1-\beta}{2 \beta}\right) \text {. } \\
& \left(\sum_{u \in V \backslash C^{*}} w\left(u, f^{*}(u)\right)+\sum_{u \in C^{*} \backslash\left\{f^{*}(z)\right\}} w\left(u, f^{*}(z)\right)+\sum_{u, v \in C^{*} \backslash\left\{f^{*}(z)\right\}} w(u, v)\right) \\
& \geq \frac{1}{2 \beta} \cdot \sum_{v \in V} w\left(S_{v}\right)-\frac{1}{2} \cdot\left(\sum_{u \in V \backslash C^{*}} w(u, z)+\sum_{u \in C^{*} \backslash\left\{f^{*}(z)\right\}} w(u, z)\right)- \\
& \left(\frac{1-\beta}{2 \beta}\right) \cdot \sum_{u, v \in C^{*} \backslash\left\{f^{*}(z)\right\}} w(u, v) \\
& \text { (by Lemma } 1, w\left(u, f^{*}(u)\right) \leq \frac{\beta}{1-\beta} \cdot w(u, z) \text { ) } \\
& =\frac{1}{2 \beta} \cdot \sum_{v \in V} w\left(S_{v}\right)-\frac{1}{2} \cdot w\left(S_{z}\right)-\left(\frac{1-\beta}{2 \beta}\right) \cdot \sum_{u, v \in C^{*} \backslash\left\{f^{*}(z)\right\}} w(u, v) \\
& \geq\left(\frac{n}{2 \beta}\right) \cdot w\left(S_{z}\right)-\frac{1}{2 \beta} \cdot w\left(S_{z}\right)-\left(\frac{1-\beta}{2 \beta}\right) \cdot \sum_{u, v \in C^{*} \backslash\left\{f^{*}(z)\right\}} w(u, v) \\
& \text { (since } \beta \leq 1 \text { ) } \\
& =\left(\frac{n-1}{2 \beta}\right) \cdot w\left(S_{z}\right)-\left(\frac{1-\beta}{2 \beta}\right) \cdot \sum_{u, v \in C^{*} \backslash\left\{f^{*}(z)\right\}} w(u, v) \\
& =\frac{r\left(S_{z}\right)}{2 \beta}-\left(\frac{1-\beta}{2 \beta}\right) \cdot \sum_{u, v \in C^{*} \backslash\left\{f^{*}(z)\right\}} w(u, v) \\
& \text { (since } \left.r\left(S_{z}\right)=(n-1) \cdot w\left(S_{z}\right)\right) \\
& \geq \frac{r\left(S_{z}\right)}{2 \beta}-\left(\frac{1-\beta}{2 \beta}\right) \cdot \sum_{u, v \in C \backslash\{z\}}(w(z, u)+w(z, v)) \quad \text { (by Claim 2) } \\
& \geq \frac{r(H)}{2 \beta} \quad \text { (by Claim 1). }
\end{aligned}
$$

This shows that $r(H) \leq 2 \beta \cdot r\left(H^{*}\right)$, and the proof is completed. 
Algorithm 2: Approximation algorithm for $\Delta_{\beta}$-SApHCR for $\beta \geq 1$

Let $U:=V$. Initially, $C=\emptyset$. Construct a spanning subgraph $H$ of $G$ by the following steps.

Step 1: Find $z=\arg \min _{v \in V} \sum_{u \in V} w(u, v)$ as the hub in $H$.

Step 2: Connect all vertices in $U \backslash\{z\}$ to $z$ as non-hubs in $H$.

Step 3: Return $H$.

Theorem 3. For any $\beta \geq 1$, there is a $2 \beta$-approximation algorithm for the Single Allocation at most $p$-Hub Center Routing problem.

Proof. It is easy to see that in time $O\left(n^{2}\right)$, Algorithm 2 returns a feasible solution of the Single Allocation at most $p$-Hub Center Routing problem. We now prove that the solution $H$ returned by Algorithm 2 satisfies the approximation ratio $2 \beta$. Let $G=(V, E, w)$ be the input graph of the Single Allocation at most $p$-Hub Center Routing problem. Let $H^{*}$ be an optimal solution of the Single Allocation at most $p$-Hub Center RoutING problem and $C^{*}$ be the set of hubs in $H^{*}$. Construct a weighted complete graph $G^{*}=\left(V, E, w^{*}\right)$ according to $H^{*}$ where $w^{*}(u, v)=\operatorname{dist}_{H^{*}}(u, v)$. Let $w\left(G^{*}\right)=\sum_{u, v} w^{*}(u, v)=r\left(H^{*}\right)$.

We use $S_{v}$ to denote the spanning star of $G$ with center $v$ and $w\left(S_{v}\right)=$ $\sum_{u \in V} w(u, v)$. Let $x=\arg \min _{v \in C^{*}}\left\{w\left(S_{v}\right)\right\}$. Define $w^{*}\left(S_{v}\right)=\sum_{u \in V} w^{*}(u, v)$ where $w^{*}(u, v)=\operatorname{dist}_{H^{*}}(u, v)$. Let $f^{*}(x)$ denote the hub adjacent to $x$ in $H^{*}$. Note that if $x$ is a hub in $H^{*}$, then $f^{*}(x)=x$.

Claim 1. $w^{*}\left(S_{x}\right)=(n-2) \cdot w\left(x, f^{*}(x)\right)+w^{*}\left(S_{f^{*}(x)}\right)$.

Proof. If $x$ is a hub in $H^{*}$, we have $f^{*}(x)=x$ and the equation holds directly. Suppose that $x$ is not a hub. We obtain that

$$
\begin{aligned}
w^{*}\left(S_{x}\right) & =\sum_{v \in V} w^{*}(x, v)=\sum_{v \in V \backslash\{x\}} w\left(x, f^{*}(x)\right)+w\left(f^{*}(x), f^{*}(v)\right)+w\left(f^{*}(v), v\right) \\
& =(n-1) \cdot w\left(x, f^{*}(x)\right)+\left(\sum_{v \in V \backslash\{x\}} w\left(f^{*}(x), f^{*}(v)\right)+w\left(f^{*}(v), v\right)\right) \\
& =(n-2) \cdot w\left(x, f^{*}(x)\right)+\left(\sum_{v \in V} w\left(f^{*}(x), f^{*}(v)\right)+w\left(f^{*}(v), v\right)\right) \\
& =(n-2) \cdot w\left(x, f^{*}(x)\right)+\left(\sum_{v \in V} w^{*}\left(f^{*}(x), v\right)\right) \\
& =(n-2) \cdot w\left(x, f^{*}(x)\right)+w^{*}\left(S_{f^{*}(x)}\right) .
\end{aligned}
$$

This completes the proof.

Claim 2. For any hub $y \in C^{*}, w^{*}\left(S_{y}\right) \geq \frac{1}{\beta} \cdot w\left(S_{y}\right)$. 
Proof. According to the $\beta$-triangle inequality, we see that $w(u, y) \leq \beta \cdot(w(u, y)+$ $\left.w\left(f^{*}(u), y\right)\right)$. We obtain that for $u \in V, w^{*}(u, y)=w\left(u, f^{*}(u)\right)+w\left(f^{*}(u), y\right) \geq$ $\frac{1}{\beta} \cdot w(u, y)$. Thus

$$
w^{*}\left(S_{y}\right)=\sum_{u \in V} w^{*}(u, y) \geq \sum_{u \in V} \frac{1}{\beta} \cdot w(u, y)=\frac{1}{\beta} \cdot \sum_{u \in V} w(u, y)=\frac{1}{\beta} \cdot w\left(S_{y}\right) .
$$

This completes the proof.

Now we prove $r(H) \leq 2 \beta \cdot r\left(H^{*}\right)$ in the following.

$$
\begin{aligned}
r\left(H^{*}\right) & =w^{*}\left(G^{*}\right)=\frac{1}{2} \cdot \sum_{v \in V} w^{*}\left(S_{v}\right) \\
& =\frac{1}{2} \cdot\left(\sum_{v \in V}\left(w^{*}\left(S_{f^{*}(v)}\right)+(n-2) \cdot w\left(v, f^{*}(v)\right)\right)\right) \quad(\text { by Claim 1) } \\
& \geq \frac{1}{2} \cdot \sum_{v \in V} w^{*}\left(S_{f^{*}(v)}\right) \geq \frac{1}{2 \beta} \cdot \sum_{v \in V} w\left(S_{f^{*}(v)}\right) \quad \text { (by Claim 2) } \\
& \geq \frac{1}{2 \beta} \cdot n \cdot w\left(S_{x}\right) \quad\left(\text { since } x=\arg \min _{v \in C^{*}}\left\{w\left(S_{v}\right)\right\}\right) \\
& \geq \frac{1}{2 \beta} \cdot(n-1) \cdot w\left(S_{z}\right) \quad\left(\operatorname{since} z=\arg \min _{v \in V}\left\{w\left(S_{v}\right)\right\}\right)=\frac{1}{2 \beta} \cdot r(H) .
\end{aligned}
$$

This shows $r(H) \leq 2 \beta \cdot r\left(H^{*}\right)$. Thus Algorithm 2 returns a solution with approximation ratio $2 \beta$.

\section{Concluding remarks}

In this paper, we have proved that the Single Allocation AT Most $p$-HuB Center Routing problem is NP-hard in $\Delta_{\beta}$-metric graphs for any $\beta>\frac{1}{2}$. For any $\beta>\frac{1}{2}$, we have given $2 \beta$-approximation algorithms. In future work, it is of interest to design approximation algorithms with better approximation ratios. Besides, it is still open whether the Single Allocation AT MOst $p$-HuB Center Routing problem is APX-hard or not. If the Single Allocation AT MOST $p$-Hub CENTER RouTING problem is APX-hard, one must prove that for any $\epsilon>0$, it is NP-hard to approximate Single Allocation AT most $p$-Hub Center Routing to a factor $c-\epsilon$ for some constant $c>1$. The other possibility is that there exists a polynomial-time approximation scheme (PTAS) for Single Allocation at most $p$-Hub Center Routing. We conjecture that there exists a PTAS for the Single Allocation at most $p$-Hub Center RouTing problem.

\section{References}

1. S. Alumur and B.Y. Kara, Network hub location problems: the state of the art, European Journal of Operational Research, 190, pp. 1V-21, 2008. 
2. T. Andreae: On the traveling salesman problem restricted to inputs satisfying a relaxed triangle inequality. Networks 38 (2001), pp. 59-67.

3. T. Andreae, H.-J. Bandelt: Performance guarantees for approximation algorithms depending on parameterized triangle inequalities. SIAM Journal on Discrete Mathematics 8 (1995), pp. 1-16.

4. M.A. Bender, C. Chekuri: Performance guarantees for the TSP with a parameterized triangle inequality. Information Processing Letters 73 (2000), pp. 17-21.

5. H.-J. Böckenhauer, D. Bongartz, J. Hromkovič, R. Klasing, G. Proietti, S. Seibert, W. Unger: On the hardness of constructing minimal 2-connected spanning subgraphs in complete graphs with sharpened triangle inequality. Proc. FSTTCS 2002, LNCS 2556, Springer 2002, pp. 59-70. Full version in Theoretical Computer Science 326 (2004), pp. 137-153.

6. H.-J. Böckenhauer, D. Bongartz, J. Hromkovič, R. Klasing, G. Proietti, S. Seibert, W. Unger: On $k$-Edge-Connectivity Problems with Sharpened Triangle Inequality. In: R. Petreschi, G. Persiano, R. Silvestri (eds.), Algorithms and Complexity, Proc. 5th Italian Conference, CIAC 2003, LNCS 2653, Springer 2003, pp. 189-200.

7. H.-J. Böckenhauer, D. Bongartz, J. Hromkovič, R. Klasing, G. Proietti, S. Seibert, W. Unger: On $k$-Connectivity Problems with Sharpened Triangle Inequality. Journal of Discrete Algorithms 6 (2008), pp. 605-617.

8. H.-J. Böckenhauer, J. Hromkovič, R. Klasing, S. Seibert, W. Unger: Approximation algorithms for the TSP with sharpened triangle inequality. Information Processing Letters 75 (2000), pp. 133-138.

9. H.-J. Böckenhauer, J. Hromkovič, R. Klasing, S. Seibert, W. Unger: Towards the Notion of Stability of Approximation for Hard Optimization Tasks and the Traveling Salesman Problem (Extended Abstract). Proc. CIAC 2000, LNCS 1767, Springer 2000, pp. 72-86. Full version in Theoretical Computer Science 285 (2002), pp. 3-24.

10. H.-J. Böckenhauer, J. Hromkovič, R. Klasing, S. Seibert, W. Unger: An Improved Lower Bound on the Approximability of Metric TSP and Approximation Algorithms for the TSP with Sharpened Triangle Inequality (Extended Abstract). Proc. STACS 2000, LNCS 1770, Springer 2000, pp. 382-394.

11. H.-J. Böckenhauer, J. Hromkovič, S. Seibert: Stability of Approximation. In: T. F. Gonzalez (ed.): Handbook of Approximation Algorithms and Metaheuristics, Chapman \& Hall/CRC, 2007, Chapter 31.

12. H.-J. Böckenhauer, S. Seibert: Improved lower bounds on the approximability of the traveling salesman problem. RAIRO - Theoretical Informatics and Applications 34 (2000), pp. 213-255.

13. J. F. Campbell, Integer programming formulations of discrete hub location problems, European Journal of Operational Research, 72 (1994), pp. 387-405.

14. J. F. Campbell and M. E. O'Kelly, Twenty-five years of hub location research, Transportation Science, 46 (2012), pp. 153-169.

15. L.-H. Chen, D.-W. Cheng, S.-Y. Hsieh, L.-J. Hung, C.-W. Lee, and B. Y. Wu, Approximation algorithms for single allocation $k$-hub center problem, Proceedings of the 33rd Workshop on Combinatorial Mathematics and Computation Theory (CMCT 2016), pp. 13-18, 2016.

16. L.-H. Chen, D.-W. Cheng, S.-Y. Hsieh, L.-J. Hung, C.-W. Lee, B. Y. Wu, Approximation algorithms for the star $k$-hub center problem in metric graphs, Proceeding of the 22nd Annual International Computing and Combinatorics Conference (COCOON 2016), LNCS 9797, pp. 222-234, 2016. 
17. L.-H. Chen, S.-Y. Hsieh, L.-J. Hung, R. Klasing, C.-W. Lee, B. Y. Wu, On the complexity of the star $p$-hub center problem with parameterized triangle inequality, The 10th International Conference on Algorithms and Complexity (CIAC 2017), LNCS 10236, pp. 152-163, 2017.

18. L.-H. Chen, S.-Y. Hsieh, L.-J. Hung, and R. Klasing, The approximability of the $p$ hub center problem with parameterized triangle inequality, Proceedings of the 23rd Annual International Computing and Combinatorics Conference (COCOON 2017), LNCS 10392, pp. 112-123, 2017.

19. L.-H. Chen, D.-W. Cheng, S.-Y. Hsieh, L.-J. Hung, R. Klasing, C.-W. Lee, and B. Y. Wu, Approximability and inapproximability of the star $p$-hub center problem with parameterized triangle inequality, Journal of Computer and System Sciences 92 (2018), pp. 92-112.

20. I. Contreras, Hub location problems, in G. Laporte, S. Nickel, and F. Saldanha da Gama, editors, Location Science, Springer, 2015.

21. A. T. Ernst, H. Hamacher, H. Jiang, M. Krishnamoorthy, and G. Woeginger, Uncapacitated single and multiple allocation $p$-hub center problems, Computer $\&$ Operations Research, 36 (2009), pp. 2230-2241.

22. J. Hromkovič: Stability of approximation algorithms and the knapsack problem. In: J. Karhumäki, H. Maurer, G. Paun, G. Rozenberg (Eds.) Jewels are Forever, Springer 1999, pp. 238-249.

23. J. Hromkovič: Algorithmics for Hard Problems - Introduction to Combinatorial Optimization, Randomization, Approximation, and Heuristics. Second Edition, Springer 2003.

24. M.R. Garey, D.S. Johnson: Computers and Intractability: A guide to the theory of NP-completeness. W. H. Freeman and Company, San Francisco, 1979.

25. M. Iwasa, H. Saito, T. Matsui, Approximation algorithms for the single allocation problem in hub-and-spoke networks and related metric labeling problems, Discrete Applied Mathematics, 157 (2009), pp. 2078-2088.

26. Y. Kuroki and T. Matsui, Approximation algorithms for hub location problems, The 9th Annual Meeting of Asian Association for Algorithms and Computation (AAAC 2016).

27. C.-W. Lin and B. Y. Wu, On the minimum routing cost clustered tree problem, Journal of Combinatorial Optimizations, 33 (2017), pp. 1106-1121.

28. N. Mladenović, J. Brimberg, P. Hansen, J.A. Moreno-Pérez, The p-median problem: a survey of metaheuristic approaches, European Journal of Operational Research 179 (2007), pp. 927-939.

29. T. Mömke, An improved approximation algorithm for the traveling salesman problem with relaxed triangle inequality. Information Processing Letters, 115 (2015), pp. $866-871$.

30. M. E. O'Kelly, A quadratic integer program for the location of interacting hub facilities. European Journal of Operational Research 32 (1987), pp. 393-404.

31. M. E. O'Kelly and H. J. Miller, Solution strategies for the single facility minimax hub location problem, Papers in Regional Science, 70 (1991), pp. 367-380.

32. R. Todosijević, D. Urošević, N. Mladenović, and S. Hanafi, A general variable neighborhood search for solving the uncapacitated $r$-allocation $p$-hub median problem, Optimization Letters 11 (2017), pp. 1109-1121.

33. B. Y. Wu, G. Lancia, V. Bafna, K.-M. Chao, R. Ravi, and C. Y. Tang, A polynomial-time approximation scheme for minimum routing cost spanning trees, SIAM Journal on Computing 29 (1999), pp. 761-778.

34. B. Y. Wu, A polynomial time approximation scheme for the two-source minimum routing cost spanning trees, Journal of Algorithms 44 (2002), pp. 359-378. 